\title{
Two women presenting aborted sudden cardiac arrest as the first event of mitral valve disease
}

\author{
Sua Kim', Jae Min Shim², Seong-Mi Park ${ }^{2}$ \\ 'Department of Critical Care Medicine, Samsung Medical Center, Sungkyunkwan University School of Medicine, Seoul;'2Division of Cardiology, Department \\ of Internal Medicine, Korea University Anam Hospital, Korea University College of Medicine, Seoul, Korea
}

Mitral valve prolapse (MVP) is a relatively common valvular heart disease and is known to have a benign course. However, a certain subtype of MVP has a pathologic prognosis and can be accompanied by malignant cardiac arrhythmia causing sudden cardiac arrest, which can be characterized by bileaflet mitral valvular thickening and prolapse and frequent premature ventricular ectopic activity upon electrocardiography. Herein, we present two patients with bileaflet mitral prolapse who survived aborted sudden cardiac arrest. These cases show a precise MVP diagnosis that may prevent a devastating life event with the unique MVP subtype.

Key Words: arrhythmia; bileaflet; mitral valvular prolapse; sudden cardiac arrest

The prevalence and cause of sudden cardiac arrest are different in young individuals compared to older subjects. Although acute coronary syndrome is the most common cause in old age, an inherited or congenital cause is more common in young people. Brugada syndrome, congenital long QT syndrome, and hypertrophic cardiomyopathy are well-known examples of such acute coronary issues [1]. However, mitral valve prolapse (MVP) is not a familiar diagnosis for sudden cardiac arrest. Within the short interval of 1 month, we experienced two cases of aborted sudden cardiac arrest combined with bileaflet MVP. These two cases show a precise diagnosis of MVP and cautious approach in managing its unique subtype, bileaflet MVP, which together may prevent a disastrous event of sudden cardiac arrest.

\section{CASE REPORTS}

\section{Case 1}

A 26-year-old woman without any medical history suddenly collapsed while she was walking. Her friends called 119, and basic life support was started in 5 minutes. An automated external defibrillator (AED) was applied three times because the cardiac rhythm showed ventricular fibrillation. Spontaneous circulation returned after 10 minutes of cardiopulmonary resuscitation (CPR). When the patient arrived at the emergency room of Korea University Anam Hospital, a 12-lead electrocardiogram (ECG) showed a sinus rhythm. There was no evidence of cerebral hemorrhage, infarction, or myocardial ischemia on brain computed tomography and 12-lead ECG. The patient was admitted to the intensive care unit (ICU) and underwent targeted temperature management. Forty-eight hours later, the patient regained

\section{Case Report}

Received: November 20, 2017

Revised: January 26, 2018

Accepted: February 20, 2018

Corresponding author

Seong-Mi Park

Division of Cardiology, Department of Internal Medicine, Korea University Anam Hospital, Korea University College of Medicine, 73 Inchon-ro, Seongbuk-gu, Seoul 02841, Korea Tel: +82-2-920-5448

Fax: +82-2-927-1478

E-mail: smparkmd@korea.ac.kr

Copyright () 2019 The Korean Society of Critical Care Medicine

This is an Open Access article distributed under the terms of Creative Attributions Non-Commercial License (http:// creativecommons.org/li-censes/by-nc/4.0/) which permits unrestricted noncommercial use, distribution, and reproduction in any medium, provided the original work is properly cited. 

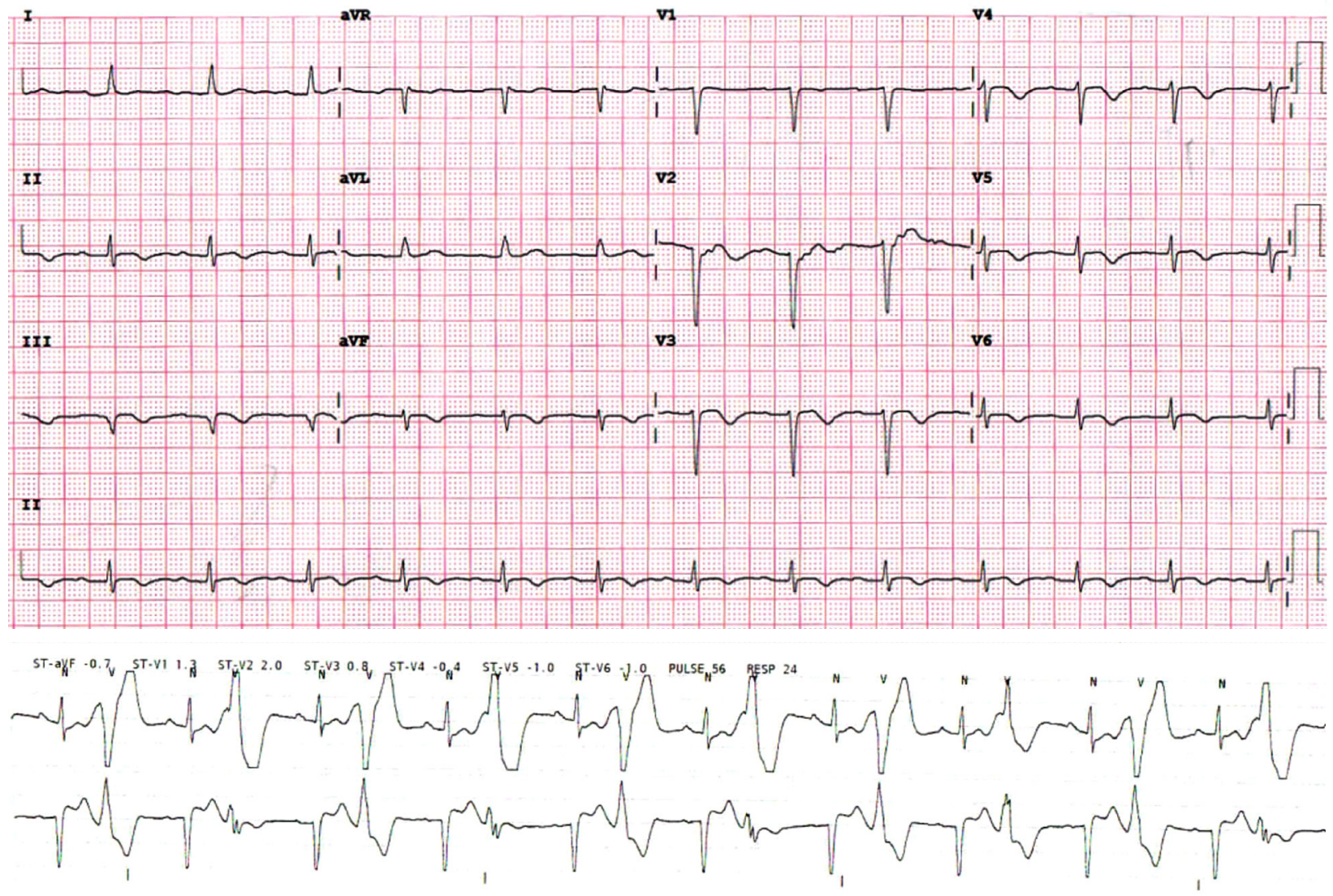

Figure 1. Electrocardiogram of case 1 presenting $T$ wave inversion of inferior leads $(A)$ and ventricular bigeminy (B).

full consciousness.

To evaluate the cause of cardiac arrest, ECG was continuously monitored via telemetry during ICU admission. Frequent premature ventricular contraction (PVC) in the bigeminy pattern and $\mathrm{T}$ wave inversion in the inferior leads were observed (Figure 1). QT was mildly prolonged up to $484 \mathrm{~ms}$. However, an epinephrine challenge test to evaluate long QT syndrome showed a negative result. Echocardiography showed bileaflet mitral prolapse and moderate mitral regurgitation. Both valvular leaflets were thickened with myxomatous change, and the posterior mitral leaflet was elongated. During systole, the mitral annulus was backwardly displaced and separated from the atrial aspect of the left ventricle (mitral annular disjunction), and the posterior mitral ring moved backward exaggeratedly and adjacent to the posterior left ventricular wall (curling). The inferomedial papillary muscle was hypertrophied, and the myocardial figure of the inferior wall looked abnormal (Figure 2). In cardiac magnetic resonance (MR) imaging, the abnormal movement of mitral annulus, hypertrophied papillary muscles, and abnormal figure of the left ventricular inferior wall were consistently observed with a focally thinned left ventricular basal inferior wall, where it was adjacent to the base of the hypertrophied papillary muscle. Although it was not very prominent, the signal intensity was inhomogeneous in that lesion upon late gadolinium imaging, which suggested the presence of damaged myocardium (Figure 3).

\section{Case 2}

A 33-year-old pregnant woman suddenly collapsed in court after complaining of palpitation. No pulse was detected, and bystander CPR was started by a civil servant who was educated in basic life support. After electrical shock administered by an AED, spontaneous circulation was returned. When the patient arrived at the hospital, she was confused but cooperative, and ECG showed a sinus rhythm with $\mathrm{T}$ wave inversion of the inferior leads and prolonged QT interval. Frequent PVCs and nonsustained ventricular tachycardia were documented on Holter ECG. However, epinephrine challenge test showed a negative result. However, upon echocardiography, moderate to severe mitral regurgitation with bileaflet MVP was found. The inferomedial papillary muscle was hypertrophied, and mitral annular disjunction and curling were also observed. 

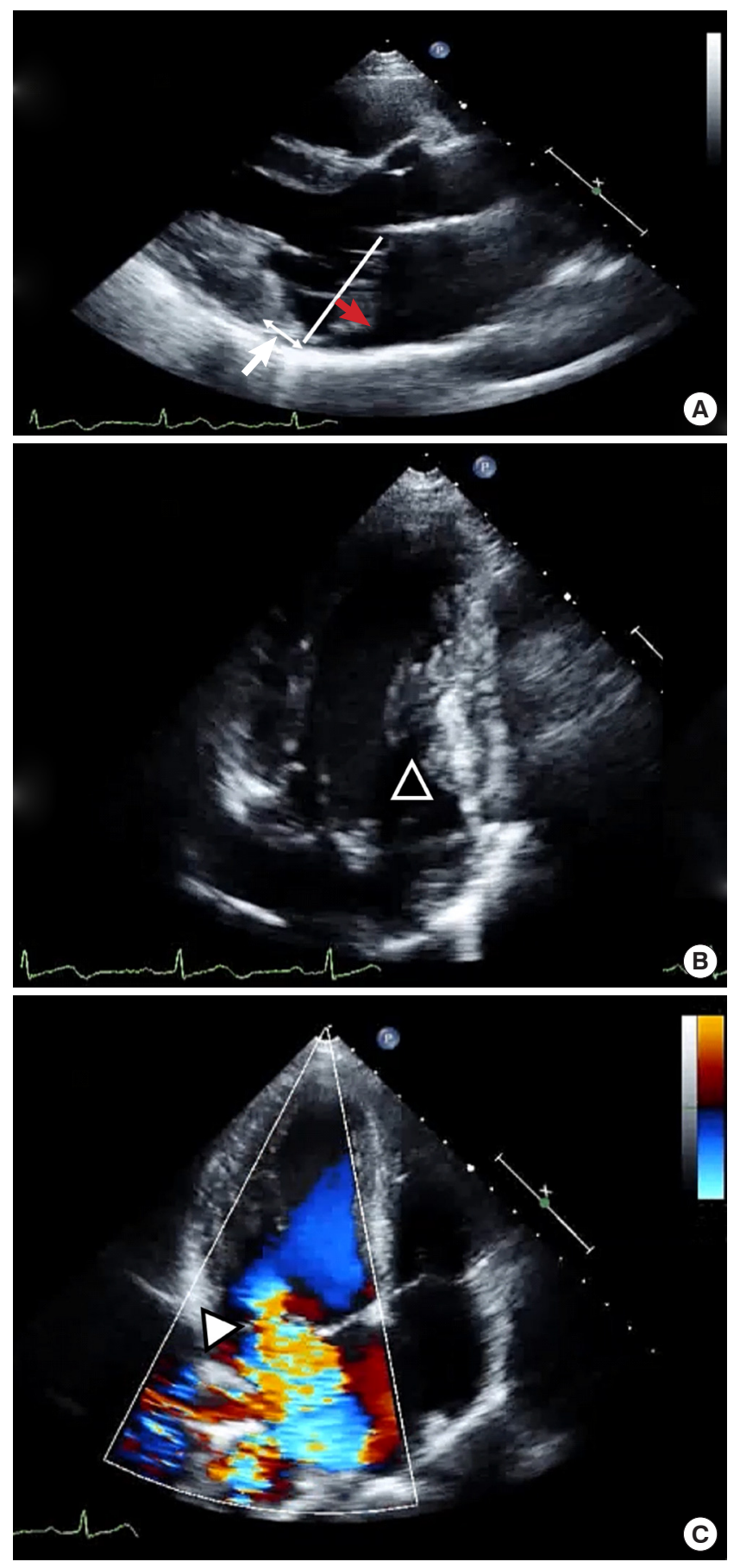

Figure 2. Echocardiographic imaging of case 1. Two-dimensional image of parasternal long axis view (A) and apical two-chamber view (B) show bileaflet mitral valvular prolapse (red arrow) and backward displacement of the mitral annular ring (white arrow). Apical two-chamber view additively shows a hypertrophied papillary muscle (arrowhead). (C) Color Doppler image of the apical fourchamber view shows moderate mitral regurgitation (arrowhead).

There was no evidence of myocardial fibrosis on cardiac MR. Both patients were diagnosed with bileaflet MVP combined with ventricular fibrillation. They were fully recovered and dis-
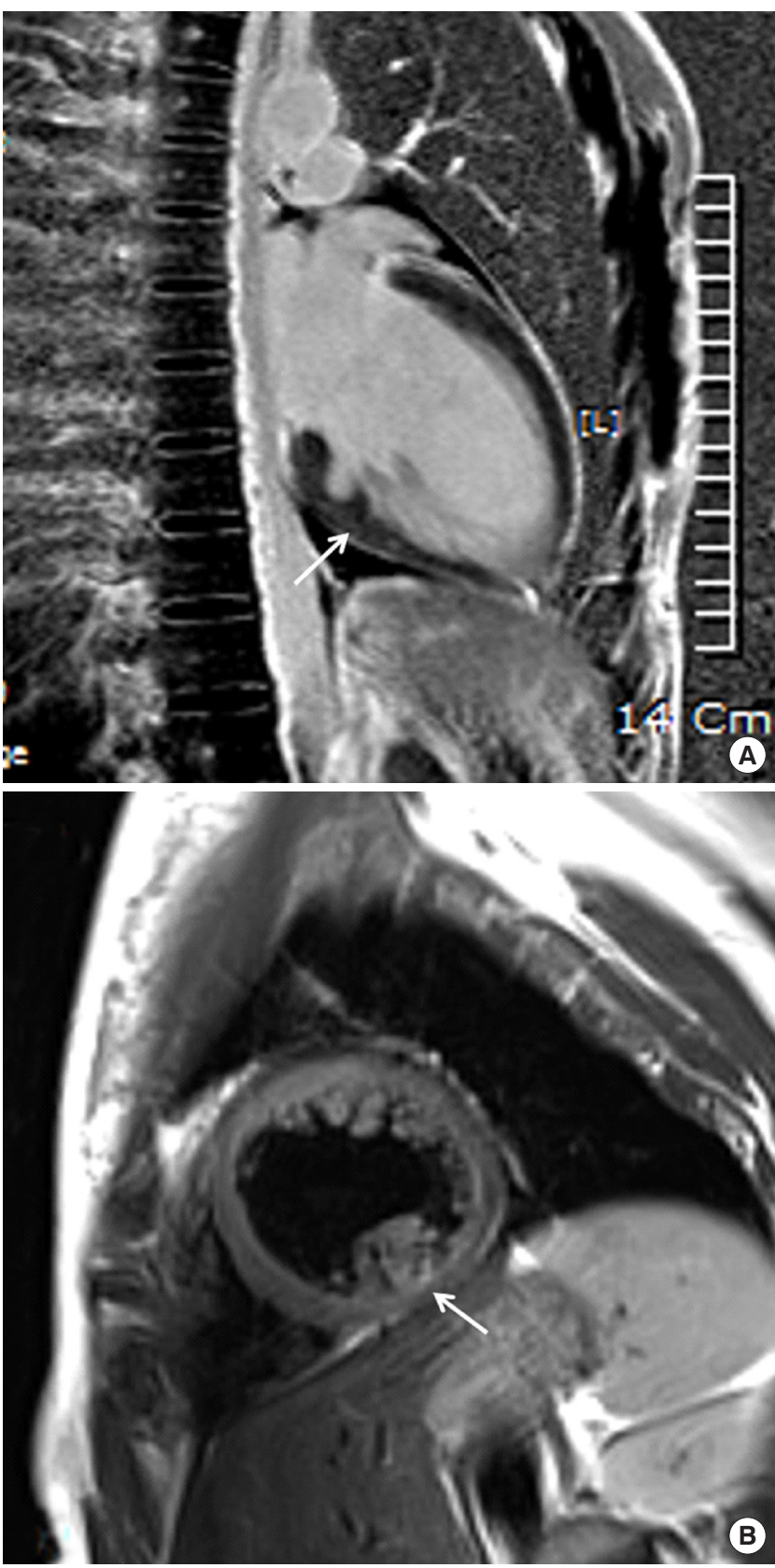

Figure 3. Cardiac magnetic resonance imaging of case 1 shows a thinned inferobasal left ventricular wall ( $A$, arrow) and inhomogenous signal intensity by late gadolinium enhancement suggesting damaged myocardium ( $\mathrm{B}$, arrow).

charged with $\beta$-blocker after placement of an implantable cardioverter defibrillator.

\section{DISCUSSION}

Within a short time interval of 1 month, two young women presented to our hospital with sudden cardiac arrest as their first sigh of cardiac disease and received ICU management. 
Interestingly, both patients presented bilateral mitral prolapse with moderate to severe regurgitation on echocardiography and $\mathrm{T}$ wave inversion of the inferior ECG leads. The patients had no family history of sudden cardiac arrest or inherited cardiac disease. Therefore, we had high suspicion of MVP as the cause and reviewed similar cases.

MVP is a common valvular heart disease. The prevalence of MVP has been reported in $2.4 \%-6 \%$ of the general population [2-4], and the natural course is benign in most cases. However, MVP is also known to cause cardiac complications such as atrial fibrillation, congestive heart failure, endocarditis, and stroke when the degree of mitral regurgitation is significant. Ventricular arrhythmia and sudden cardiac arrest have been reported as a result of MVP in a very small percentage of cases [5]. Interest has been focused recently on such patients with MVP and ventricular arrhythmia as the cause of sudden cardiac arrest $[6,7]$.

It is difficult to know the exact prevalence of pathologic arrhythmic events among MVP patients because most of the patients show a benign course and are not diagnosed before symptom presentation. Therefore, sudden cardiac arrest can occur as the first presentation of the disease. Cardiac arrest has been reported to occur at $0.2 \%-0.4 \%$ per year in patients with MVP [4]. These patients occupy $2 \%$ of out-of-hospital cardiac arrest survivors and $7 \%$ of sudden cardiac arrest survivors younger than 40 years $[5,6]$. Occurrence in females was much more common [8], and MVP causing a pathologic arrhythmic event typically showed bileaflet prolapse or otherwise involved the posterior leaflet rather than the anterior leaflet. Thickening of the involved leaflet with myxomatous change and elongation of papillary muscle and/or chordae were also observed. Structural changes of the mitral apparatus combined with hypermobile mitral annular movement cause significant mitral regurgitation and structural changes of the left ventricle. ECG typically shows frequent PVCs and T wave inversion of inferior leads [5]. Studies using cardiac MR imaging showed focal hypertrophy or fibrosis of the inferobasal wall of the left ventricle or papillary muscles [6], which can become the substrate of ventricular arrhythmia. The prevalence of PVC and ventricular tachycardia in survivors of outof-hospital cardiac arrest were significantly greater in patients with bileaflet MVP than in patients with unileaflet MVP, and their origin of arrhythmia was the papillary muscle [5].

Sudden cardiac arrest is a rare but devastating event to the patients themselves and their family members. Even upon survival, the resultant complication may cause disability, which increases social cost and family burden, especially when af- flicted individuals are young. Therefore, control of known risk factors of sudden cardiac arrest in the young population is very important. Currently in Korea, the diagnosis of MVP is increasing with active general medical examination programs. The selection of patients at higher risk of sudden cardiac arrest among MVP patients and their proper management will contribute to reducing the rare but serious event.

\section{CONFLICT OF INTEREST}

No potential conflict of interest relevant to this article was reported.

\section{ORCID}

Sua Kim

Jae Min Shim

https://orcid.org/0000-0003-3513-7261

Seong-Mi Park https://orcid.org/0000-0001-8251-1522 https://orcid.org/0000-0002-6710-685X

\section{AUTHOR CONTRIBUTIONS}

Conceptualization: SMP. Data curation \& Methodology: JMS. Project administration: SMP. Visualization \& Writing - original draft: SK. Writing - review \& editing: SMP.

\section{REFERENCES}

1. Batra AS, Balaji S. Prevalence and spectrum diseases predisposing to sudden cardiac death: are they the same for both the athlete and the nonathlete? Pediatr Cardiol 2012;33:379-86.

2. Angella FR, Lewis JF. Mitral valve prolapse: gender differences in evaluation and management. Cardiol Rev 1999;7:161-8.

3. Kligfield P, Levy D, Devereux RB, Savage DD. Arrhythmias and sudden death in mitral valve prolapse. Am Heart J 1987;113: 1298-307.

4. Nishimura RA, McGoon MD, Shub C, Miller FA Jr, Ilstrup DM, Tajik AJ. Echocardiographically documented mitral-valve prolapse: long-term follow-up of 237 patients. N Engl J Med 1985; 313:1305-9.

5. Sriram CS, Syed FF, Ferguson ME, Johnson JN, Enriquez-Sarano $\mathrm{M}$, Cetta $\mathrm{F}$, et al. Malignant bileaflet mitral valve prolapse syndrome in patients with otherwise idiopathic out-of-hospital cardiac arrest. J Am Coll Cardiol 2013;62:222-30.

6. Basso C, Perazzolo Marra M, Rizzo S, De Lazzari M, Giorgi B, Cipriani A, et al. Arrhythmic mitral valve prolapse and sudden cardiac death. Circulation 2015;132:556-66.

7. Perazzolo Marra M, Basso C, De Lazzari M, Rizzo S, Cipriani 
A, Giorgi B, et al. Morphofunctional abnormalities of mitral annulus and arrhythmic mitral valve prolapse. Circ Cardiovasc Imaging 2016;9:e005030.
8. Anders S, Said S, Schulz F, Püschel K. Mitral valve prolapse syndrome as cause of sudden death in young adults. Forensic Sci Int 2007;171:127-30. 